\title{
Two supernova remnants of low radio surface brightness discovered in the Canadian Galactic Plane Survey
}

\author{
R. Kothes ${ }^{1}$, T. L. Landecker ${ }^{1}$, T. Foster ${ }^{2}$, and D. A. Leahy ${ }^{3}$ \\ 1 National Research Council of Canada, Herzberg Institute of Astrophysics, Dominion Radio Astrophysical \\ Observatory, PO Box 248, Penticton, British Columbia, V2A 6K3, Canada \\ e-mail: tom.landecker@nrc.ca \\ 2 Department of Physics, University of Alberta, Edmonton, Alberta, T6G 2J1, Canada \\ e-mail: tfoster@phys.ualberta.ca \\ 3 Department of Physics and Astronomy, University of Calgary, Calgary, Alberta, T2N 1N4, Canada \\ e-mail: leahy@iras.ucalgary.ca
}

Received 20 March 2001 / Accepted 2 July 2001

\begin{abstract}
Two new supernova remnants (SNRs), G85.4+0.7 and G85.9-0.6, superimposed on the radio source W 80, have been discovered in the radio continuum data from the Canadian Galactic Plane Survey(CGPS). Both SNRs consist of a thin incomplete radio shell surrounding a weak, extended X-ray source. G85.4+0.7 has a thin non-thermal shell of diameter $\approx 0.4^{\circ}$ lying within a thermal shell whose diameter is $\approx 0.6^{\circ}$. Its radio surface brightness at $1 \mathrm{GHz}$ is $\Sigma_{1 \mathrm{GHz}} \leq 1 \times 10^{-22}$ Watt $\mathrm{m}^{-2} \mathrm{~Hz}^{-1} \mathrm{sr}^{-1}$. It is located within a large $\mathrm{H}$ i bubble, whose systemic velocity, $\mathrm{v}_{\mathrm{LSR}}=-12 \mathrm{~km} \mathrm{~s}^{-1}$, implies a distance of $3.8 \mathrm{kpc}$. Two B1 stars detected within this bubble are most likely part of the OB association which formed it. The diameter of the H i bubble is about 100 pc. The SNR has a diameter of about $30 \mathrm{pc}$ and probably is the result of a type II explosion of an early B star $\approx 6300$ years ago. G85.9-0.6 has a radio surface brightness of $\Sigma_{1 \mathrm{GHz}} \leq 2 \times 10^{-22} \mathrm{Watt} \mathrm{m}^{-2} \mathrm{~Hz}^{-1} \mathrm{sr}^{-1}$. No H I features corresponding to the SNR have been detected. This, and the low radio and X-ray brightnesses, suggest expansion in a low-density medium. The SNR may lie in the low-density region between the local and Perseus spiral arms, at a distance of about $5 \mathrm{kpc}$. Its diameter would then be $\sim 35 \mathrm{pc}$.
\end{abstract}

Key words. ISM: supernova remnant - ISM: individual object: G85.4+0.7 - ISM: individual object: G85.q+0.6 radio continuum: ISM - X-rays: ISM - ISM: bubbles

\section{Introduction}

Our current view of supernova remnants (SNRs) has been shaped by the study of the radio bright objects, all of which have probably now been detected. On the other hand, the population of SNRs in the Galaxy is likely to be dominated by objects with low surface brightness. We expect 80 to $90 \%$ of Galactic supernova explosions to be of type Ib/c or II (van den Bergh \& McClure 1994). These are the explosions of massive progenitor stars, which generally create stellar wind bubbles around them. An explosion in this environment can be expected to lead to a SNR with very low radio surface brightness because of the low ambient density. We can also anticipate the presence of confusing thermal emission from gas ionized by the progenitor or other associated stars which have not yet exploded. Recently several large SNRs and SNR candidates of low radio surface brightness have been discovered using

Send offprint requests to: $\mathrm{R}$. Kothes,

e-mail: roland.kothes@nrc.ca single-antenna telescopes (see Green 2000 for references). The discovery of smaller SNRs, however, requires a new survey with high sensitivity and excellent angular resolution at low radio frequencies, to give high sensitivity to faint, filamentary, non-thermal emission. The Canadian Galactic Plane Survey (CGPS) meets these requirements. A significant feature of the CGPS is the incorporation of single-antenna data to retain sensitivity to the largest structures.

A second important SNR signature is thermal X-ray emission from shock-heated gas. The ROSAT All-Sky Survey has revealed a number of new SNRs with bright X-ray emission and weak or no radio emission (e.g. Pfeffermann et al. 1991; Greiner et al. 1994; Busser et al. 1996). It is also a valuable resource in confirming the detection of newly discovered SNRs.

In this paper we describe two new SNRs of very low radio surface brightness, discovered in the CGPS data and confirmed by the ROSAT All-Sky Survey. 


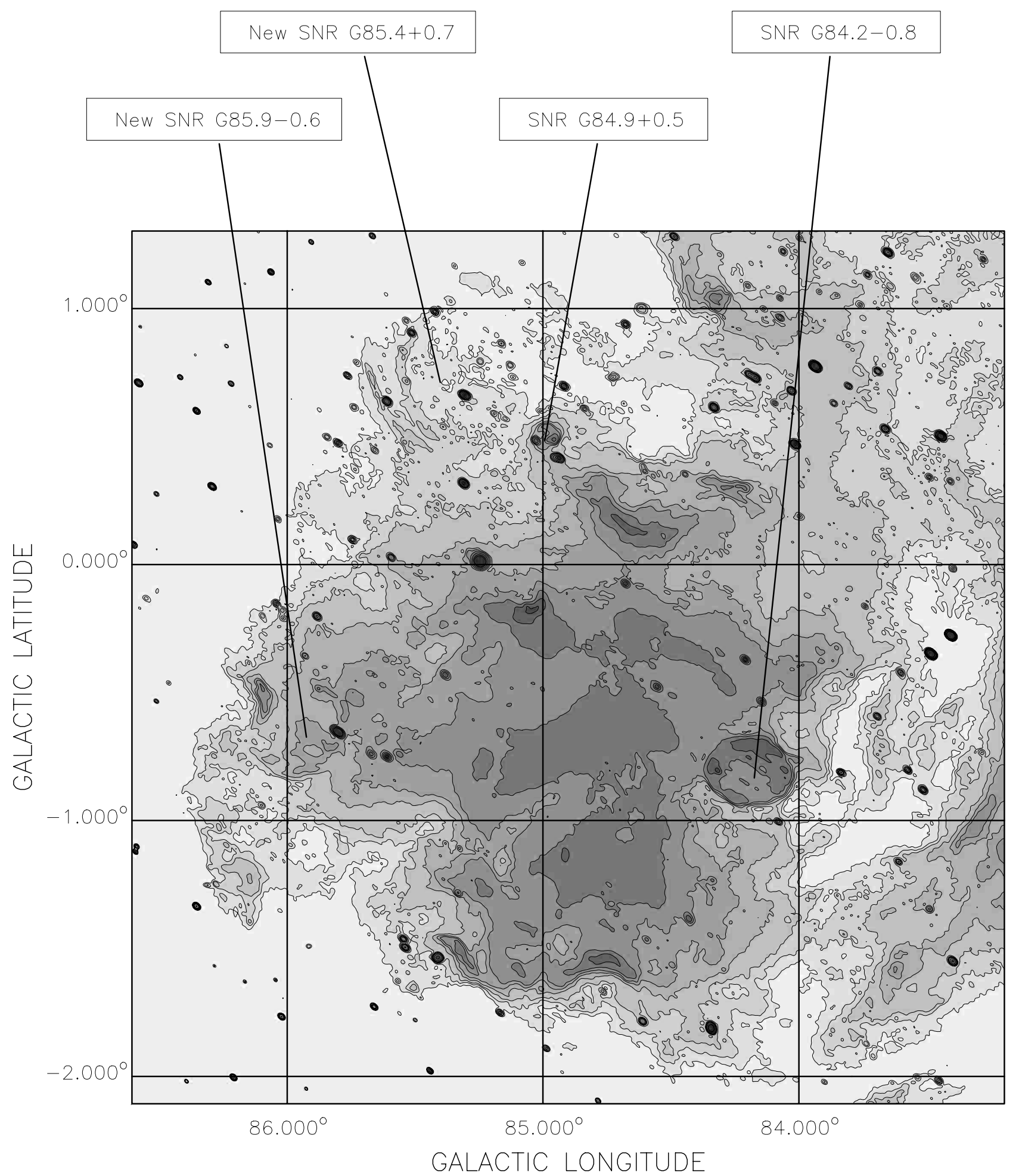

Fig. 1. Greyscale image of the W 80 region at $1420 \mathrm{MHz}$ taken from the Canadian Galactic Plane Survey. The newly discovered SNRs are marked, as are previously known SNRs. Angular resolution is approximately $49^{\prime \prime} \times 74^{\prime \prime}$. Contours are at $9,9.5,10$, $11,12,13,15,20,30$, and $40 \mathrm{~K}$.

\section{Observations and data reduction}

\subsection{Radio observations}

The CGPS is described in detail by Taylor et al. (2001). In carrying out the research presented here we have used those parts of the CGPS database which derive from observations with the DRAO Synthesis Telescope (Landecker et al. 2000). The characteristics of the survey relevant to the data presented here are listed in Table 1. The survey area is covered by observations of individual fields whose centres lie on a hexagonal grid of spacing $112^{\prime}$. 
Table 1. Survey characteristics for the area shown in Fig. 1.

\begin{tabular}{|c|c|}
\hline Operating frequencies: & $\begin{array}{l}1420 \mathrm{MHz} \\
408 \mathrm{MHz}\end{array}$ \\
\hline Angular resolution: & $\begin{array}{l}49^{\prime \prime} \times 1^{\prime} 14^{\prime \prime} \\
2.8^{\prime} \times 4.4^{\prime}\end{array}$ \\
\hline 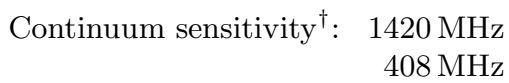 & $\begin{array}{l}0.5 \mathrm{mJy} / \text { beam rms } \\
6 \mathrm{mJy} / \text { beam } \mathrm{rms}\end{array}$ \\
\hline Spectrometer frequency coverage: & $1.0 \mathrm{MHz}$ \\
\hline Number of spectrometer channels: & 256 \\
\hline Velocity coverage for $\mathrm{HI}$ : & +60 to $-165 \mathrm{~km} \mathrm{~s}^{-1}$ \\
\hline Channel separation: & $0.824 \mathrm{~km} \mathrm{~s}^{-1}$ \\
\hline Channel width: & $1.32 \mathrm{~km} \mathrm{~s}^{-1}$ \\
\hline Noise on 1-channel spectral map: & $2.4 \mathrm{~K}$ \\
\hline
\end{tabular}

$\dagger$ Sensitivity is limited by confusion due to complex extended structure in the Cygnus region, not by thermal noise.

Angular resolution varies as cosec(declination) and therefore changes slowly across the final mosaics. The values given in Table 1 are characteristics at the centre of the image shown in Fig. 1. Before assembly into a mosaic the data for the individual fields are carefully processed to remove artefacts and to obtain the highest dynamic range, using the routines described by Willis (1999).

Accurate representation of all structures to the largest scales is assured by incorporating data from large single antennas with data from the Synthesis Telescope, after suitable filtering in the Fourier domain. Continuum data are derived from the 408-MHz All-Sky survey of Haslam et al. (1982), and from the $1.4 \mathrm{GHz}$ Effelsberg survey (Reich et al. 1997). Single-antenna H I data are obtained from a survey of the CGPS region made with the DRAO 26-m Telescope (Higgs \& Tapping 2000).

\subsection{Optical observations}

Optical observations were made of a field around one of the candidate SNRs, G85.4+0.7, at the Devon Astronomical Observatory of the University of Alberta on 2000 November 19. Absolute photometric measurements were made of 45 stars $\left(8.9<m_{v}<14.2\right)$ within a $25^{\prime}$ field centred on $\alpha(\mathrm{J} 2000)=20^{\mathrm{h}} 50^{\mathrm{m}} 45^{\mathrm{s}}, \delta(\mathrm{J} 2000)=45^{\mathrm{o}} 15^{\prime} \cdot 0$. Data were obtained with a medium-format CCD camera held at the $f / 3$ prime focus of a $0.5-\mathrm{m}$ mirror. The camera uses a back-illuminated, AR coated TK512 CCD chip $(512 \times 512$ pixels $)$, producing a field of $27.1^{\prime}$ at a scale of $3.2^{\prime \prime} /$ pixel. The chip responds linearly to better than $0.5 \%$ over a range of 10-65 200 Analog-Digital Units (ADU). This sensitive imaging system has been used to produce optical images of faint, extended radio sources found in the CGPS (e.g. H II regions and SNRs: Foster \& Routledge 2001), and also supports a program of precision differential and absolute photometry on variable stars.

All observations were carried out using $U, B$, and $V$ filters, whose characteristics are listed in Table 2. Ten nearby standard stars were observed over a range of 1 to 2 airmasses. These standards were selected from Oja (1996), and Landolt (1983), and were chosen for their
Table 2. Filter parameters for the Devon Astronomical Observatory instrument, and night-sky extinction coefficients derived from $U B V$ observations of 2000 November 19.

\begin{tabular}{|c|c|c|c|}
\hline Filter & $\lambda_{\text {Central }}( \pm 1 \mathrm{n}$ & $F l$ & $H M( \pm 0.5 \mathrm{nn}$ \\
\hline $\bar{U}$ & 365 & & 70 \\
\hline$B$ & 440 & & 100 \\
\hline$V$ & 550 & & 90 \\
\hline \multicolumn{2}{|c|}{ Extinction Symbol } & Value & "Uncertainty \\
\hline \multicolumn{2}{|c|}{$k_{v}^{\prime}$} & 0.206 & 0.028 \\
\hline \multicolumn{2}{|r|}{$k_{b-v}^{\prime}$} & 0.209 & 0.032 \\
\hline \multicolumn{2}{|r|}{$k_{u-b}^{\prime}$} & 0.357 & 0.053 \\
\hline \multicolumn{2}{|r|}{$k_{b-v}^{\prime \prime \prime}$} & 0.120 & 0.023 \\
\hline
\end{tabular}

proximity to the target stars. A wide range of brightness and colours was included in the list, with a magnitude range $7.6 \leq m_{v} \leq 13.1$, and colour index coverage $-0.317 \leq(B-V) \leq 1.147$.

Observing and data reduction procedures necessary for precision CCD photometry were followed strictly. Mean zero, dark, and flat calibration frames were made from many individuals, and noise characteristics of the CCD were measured and found to match well with average values for the system (Gain $=4.6 \mathrm{e}^{-} \mathrm{ADU}^{-1}$, Readnoise $=$ $\left.40 \mathrm{e}^{-} \pm 2 \mathrm{e}^{-}\right)$. Since the Devon telescope produces extremely sharp stellar images (typically $1.5<F W H M<$ 2 pixels), undersampling was a concern. Observations were made with the system slightly defocussed, and the resulting stellar images had a reasonable 3.5-pixel FWHM. All reductions and aperture photometry were performed with IRAF software.

The first and second order extinction values for the night, $\left(k_{v}^{\prime}, k_{(b-v)}^{\prime}, k_{(u-b)}^{\prime}, k_{(b-v)}^{\prime \prime}\right)$, were derived using two early A-type stars from the standard series, and were applied to all reductions (Table 2). The transformation coefficients and zero-point values for the system were obtained from least-squares solutions to transformation equations, as outlined in Henden et al. (1982). Mean and average absolute differences between transformed and published values for the standard stars were -0.066 and 0.073 in $V, 0.021$ and 0.057 for $(B-V)$, and 0.062 and 0.182 for $(U-B)$ colour transformations. Coefficients of correlation between transformed and published values were $C_{V}=1.014 \pm 0.019, C_{(B-V)}=0.976 \pm 0.062$, and $C_{(U-B)}=0.800 \pm 0.110$.

\section{Results}

Two new SNRs superimposed on the radio source W 80 have been discovered. Parts of this source correspond to the well-known optical features, the North America Nebula and the Pelican Nebula. Figure 1 shows the $1420 \mathrm{MHz}$ continuum emission from the entire region. W 80 is about $3^{\circ} \times 3^{\circ}$ in extent, and displays structures on all scales down to the resolution of our observations, about $1^{\prime}$. The two newly discovered SNRs are indicated in the image, as are the only other SNRs known in this region. The latter were identified through their high 

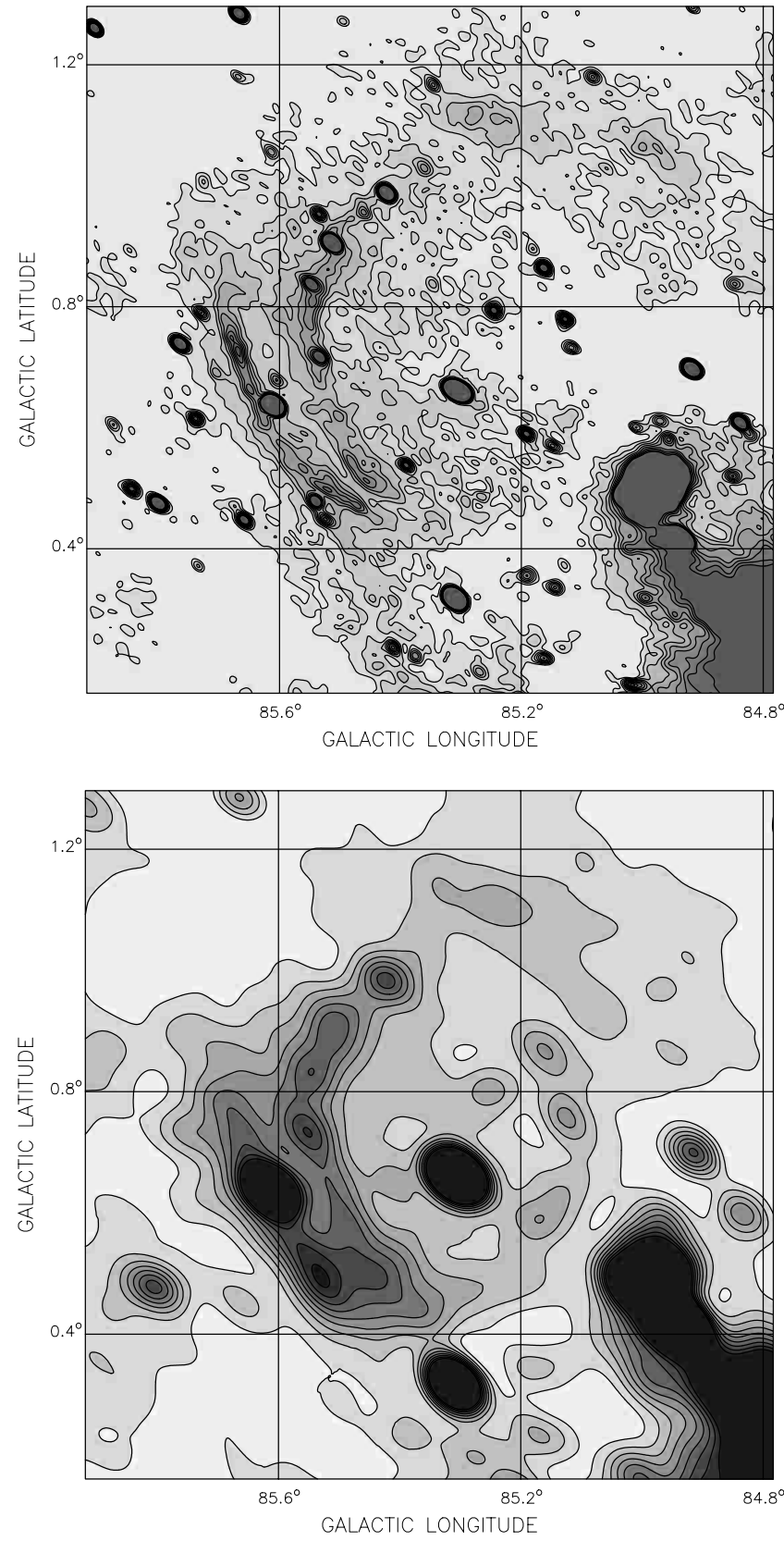

Fig. 2. Grayscale images of the new SNR G85.4+0.7 at $1420 \mathrm{MHz}$ (top) and $408 \mathrm{MHz}$ (bottom) taken from the Canadian Galactic Plane Survey. Contour levels are from $0 \mathrm{mK}$ to $1600 \mathrm{mK}$ in steps of $200 \mathrm{mK}$ and from $0 \mathrm{~K}$ to $24 \mathrm{~K}$ in steps of $3 \mathrm{~K}$ for 1420 and $408 \mathrm{MHz}$ respectively. A twisted plane background has been subtracted from each image.

surface-brightness at low radio frequencies. G84.2-0.8 was found by Matthews et al. (1977) with the Westerbork Synthesis Radio Telescope at $610 \mathrm{MHz}$. G84.9+0.5 was discovered by Taylor et al. (1992) in survey data from the same telescope at $327 \mathrm{MHz}$ used in conjunction with the $4850 \mathrm{MHz}$ Greenbank survey (Condon et al. 1989).

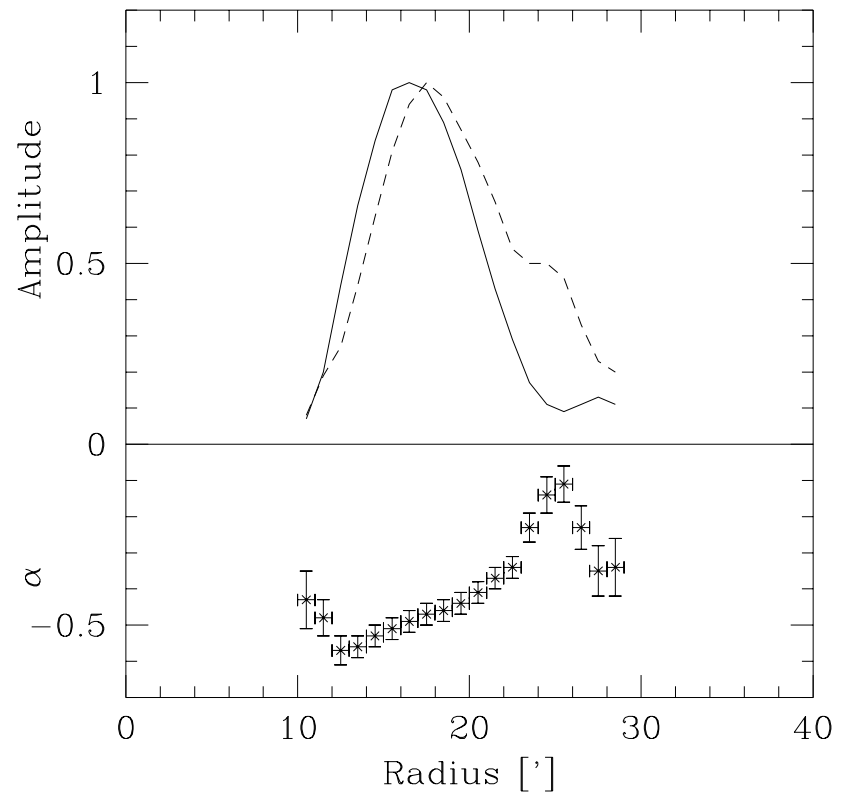

Fig. 3. Average radial profiles and the spectral behaviour of the new SNR G85.4+0.7 as a function of radius. The profiles were normalized to their peak values. The solid line represents a profile calculated for $100 \mathrm{MHz}$ and the dashed line a profile at $10 \mathrm{GHz}$ (see text).

\subsection{Radio continuum data}

\subsubsection{G85.4+0.7}

Figure 2 shows images of G85.4+0.7 at 1420 and $408 \mathrm{MHz}$. To create these images we removed the smooth background emission from W 80 by subtracting a twisted plane. This twisted plane was derived from a least square fit to all data points below a certain level in the area displayed in Fig. 2.

The source consists of two distinct shells. A comparison of the data at the two frequencies indicates that the inner shell is non-thermal while the outer shell is thermal. To get more accurate spectra we need observations at more than two frequencies or we need data over a wider frequency range. We were able to detect it in other surveys, including the Westerbork Survey at $325 \mathrm{MHz}$ (Rengelink et al. 1997), the Effelsberg Survey at $2695 \mathrm{MHz}$ (Fürst et al. 1990), and the Greenbank Survey at $4850 \mathrm{MHz}$ (Condon et al. 1994). We convolved all observations to the resolution of the Effelsberg Survey $\left(4.4^{\prime}\right)$. Since most of the surveys were made with interferometers, most extended emission is missing. To get comparable coverage we applied the "background filtering technique" of Sofue \& Reich (1979) to all data to remove extraneous smooth background emission.

After removing the stronger point sources we calculated average profiles over the eastern half of the source at all frequencies. The centre position was assumed to be at $l=85^{\circ} 22^{\prime}, b=0^{\circ} 47^{\prime}$. From these radial profiles we calculated the spectral index $\alpha$ as a function of radius. The result is displayed in Fig. 3 together with calculated profiles at $100 \mathrm{MHz}$ and $10 \mathrm{GHz}$. Even though both shells are 
smoothed together due to the low resolution it is obvious that the inner shell shows a non-thermal spectrum with a spectral index of about $\alpha=-0.5$ and the outer shell a thermal spectrum.

\subsubsection{G85.9-0.6}

Figure 4 shows images of G85.9-0.6 at 1420 and $408 \mathrm{MHz}$. From these two images, we deduce that the bright region at $(l, b)=\left(86.1^{\circ},-0.5^{\circ}\right)$ displays a thermal spectrum, while the semi-circular region at $(l, b)=\left(85.9^{\circ},-0.6^{\circ}\right)$ is non-thermal. Clearly both sources show the same radio surface brightness at $408 \mathrm{MHz}$ but at $1420 \mathrm{MHz}$ the thermal shell is much brighter. It has not been possible to identify G85.9-0.6 in the other radio data consulted. The CGPS data are superior to most of the other available radio surveys either in angular resolution, in completeness of $(u, v)$-plane coverage (because of the incorporation of single-antenna data), or in sensitivity.

Our conclusions about the nature of the sources are strengthened by comparison with optical data. Figure 5 shows a comparison of optical data from the Digitized Sky Survey, in greyscale representation, with our $1420 \mathrm{MHz}$ data. There is a very good correspondence between most radio and optical features, suggesting that most of the emission in the area is thermal. The exception is the semicircular structure centred at $(l, b)=\left(85.9^{\circ},-0.6^{\circ}\right)$ which we have identified as non-thermal emission. Obscuration in this direction appears to be low.

G85.9-0.6 consists of a thin shell of low radio surface brightness, indicating an expansion in a low density medium. The centre of the SNR is at $(l, b)=\left(85.9^{\circ},-0.6^{\circ}\right)$ and it has a radius of $\sim 12^{\prime}$.

Both objects are located at the edge of the thermal emission from W 80 which makes it almost impossible to derive meaningful flux density values. W 80 contributes an unknown diffuse background to the emission of the SNRs and there are nearby thermal filaments which make it difficult to outline the boundaries of the objects. There are also many point-like sources in the direction of the SNRs, especially towards G85.4+0.7. We derived upper limits for the flux values at $1420 \mathrm{MHz}$ which are $\approx 450 \mathrm{mJy}$ for G85.4+0.7 and $\approx 900 \mathrm{mJy}$ for G85.9-0.6. Assuming a spectral index of $\alpha=-0.5$ and a diameter of $24^{\prime}$ for both SNRs we get upper limits for the radio surface brightness at $1 \mathrm{GHz}$ of $\Sigma_{1 \mathrm{GHz}} \approx$ $1 \times 10^{-22}$ Watt $\mathrm{m}^{-2} \mathrm{~Hz}^{-1} \mathrm{sr}^{-1}$ for the SNR G85.4+0.7 and $\Sigma_{1 \mathrm{GHz}} \approx 2 \times 10^{-22}$ Watt $\mathrm{m}^{-2} \mathrm{~Hz}^{-1} \mathrm{sr}^{-1}$ for G85.90.6. There are only 2 SNRs listed in Green's supernova remnant catalogue (Green 2000) which have lower radio surface brightnesses than $10^{-22}$ Watt $\mathrm{m}^{-2} \mathrm{~Hz}^{-1} \mathrm{sr}^{-1}$. These are the SNR G182.4+4.3 (Kothes et al. 1998) and G156.2+5.7 (Reich et al. 1991). Both are very large anticentre objects. G182.4+4.3 is a young remnant expanding in a low density medium similar to G85.9-0.6 and G156.2+5.7 is expanding inside a stellar wind bubble like G85.4+0.7. Both G182.4+4.3 and G156.2+5.7 were
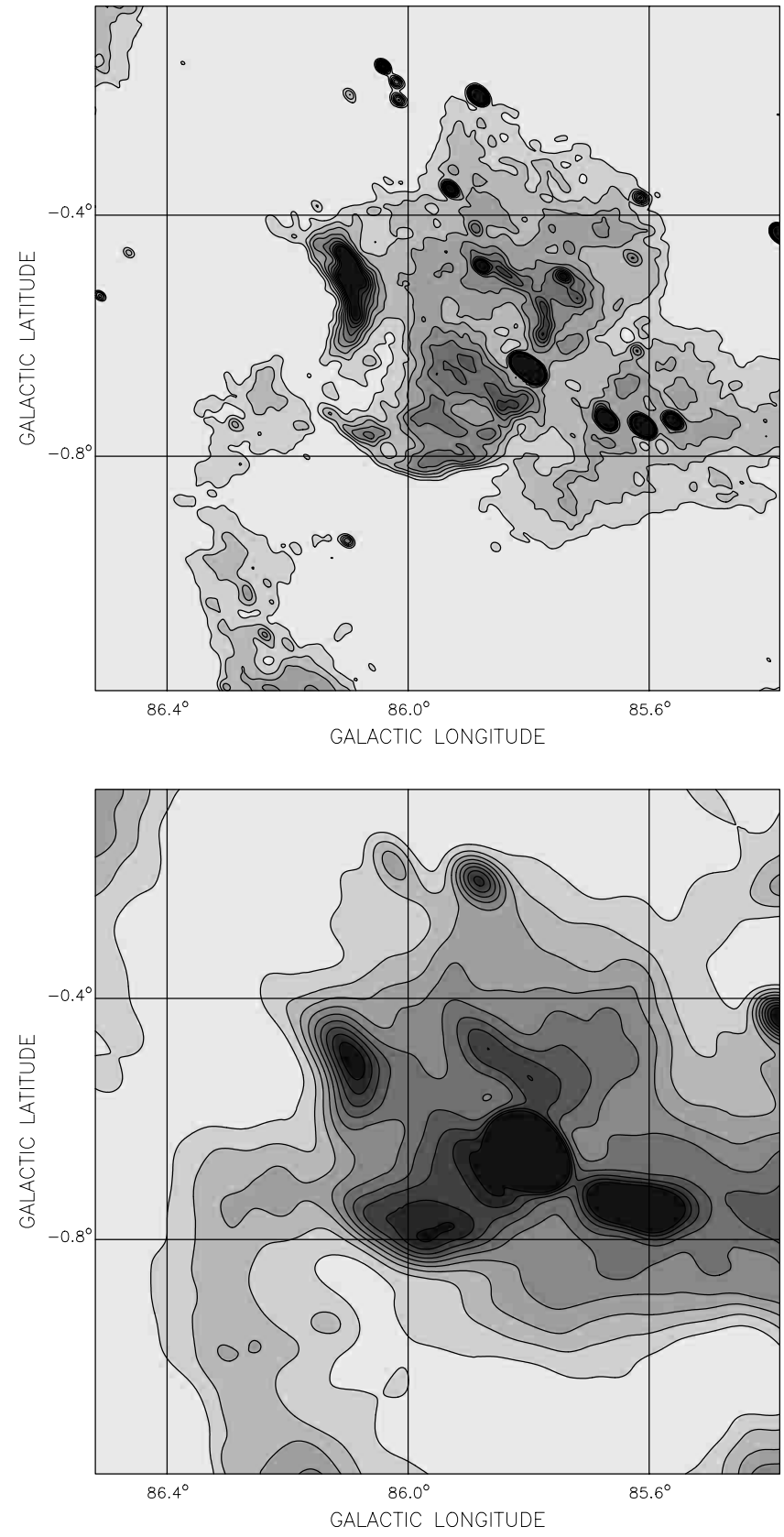

Fig. 4. Grayscale images of the new SNR G85.9-0.6 at $1420 \mathrm{MHz}$ (top) and $408 \mathrm{MHz}$ (bottom) taken from the Canadian Galactic Plane Survey. Contour levels are from 0.4 to $3.6 \mathrm{~K}$ in steps of $0.4 \mathrm{~K}$ and from 6 to $48 \mathrm{~K}$ in steps of $6 \mathrm{~K}$ at $1420 \mathrm{MHz}$ and $408 \mathrm{MHz}$ respectively. A twisted plane background has been subtracted from each image.

discovered in regions towards the anti-centre at high latitudes where there is little or no confusion with other extended structure. This is in marked contrast to the situation we are dealing with in the present work. Indeed, a major conclusion of this paper is that a survey with the characteristics of the CGPS, excellent angular resolution at low radio frequencies, is needed to identify faint extended SNRs amongst the confusing thermal emission near the Galactic plane in the inner parts of the Galaxy. 


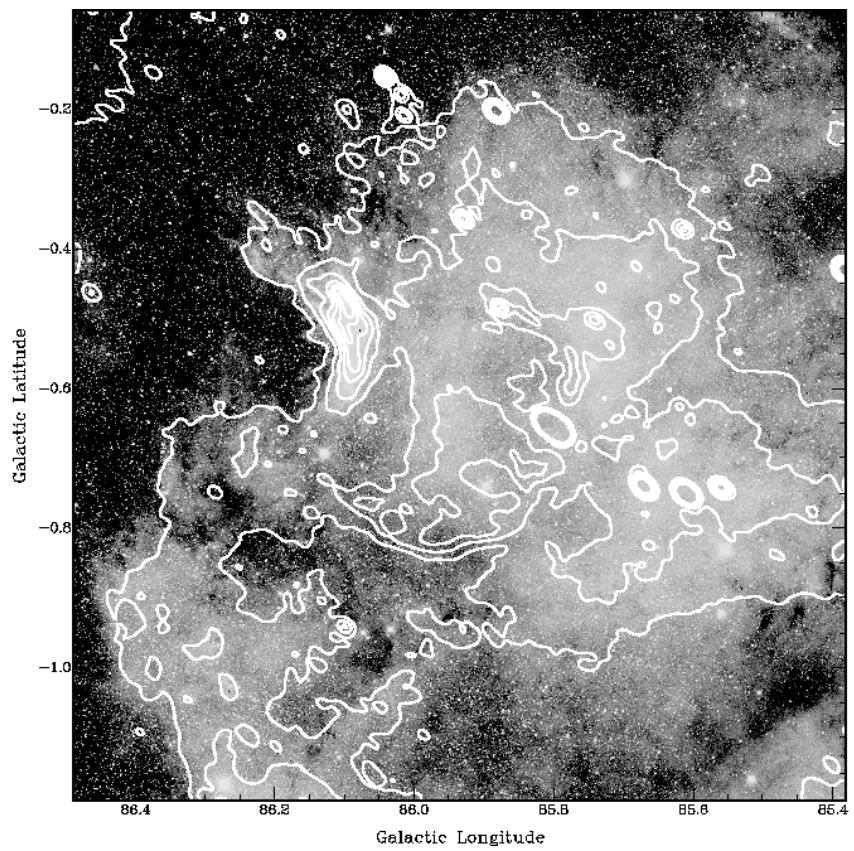

Fig. 5. Optical image of the area around the new SNR G85.90.6 taken from the Digitized Sky Survey. The white contours represent the radio continuum emission at $1420 \mathrm{MHz}$.

\subsection{Comparison with ROSAT data}

Inspection of the ROSAT All-Sky data (http://www. xray.mpe.mpg.de/cgi-bin/rosat/rosat-survey) reveals that both G85.4+0.7 and G85.9-0.6 are X-ray sources. Both sources are detectable in the highest energy band ( 0.5 to $2.4 \mathrm{keV}$ ), they are barely detectable in the mid-energy band, and are undetectable in the lowest energy band. Figure 6 shows the radio images in grayscale rendition with superimposed representation of X-ray emission for both SNRs. For this image the data from the highest energy band of the ROSAT All-Sky survey have been smoothed to $20^{\prime}$. The X-ray emission lies within the non-thermal filaments, but we must emphasize that the $\mathrm{X}$-ray emission is very weak. Because of the low intensity, it is not possible to derive X-ray spectra, and hence no information is available on temperatures. However, since we see the thermal X-ray sources only in the hard energy band, either the temperature of the SNRs is very high, implying young remnants, or interstellar absorption is very high in this direction. The latter is definitely the case for G85.4+0.7 (see Sect. 3.4).

\subsection{HI data}

\subsubsection{The $\mathrm{H}$ I environment of $\mathrm{G} 85.4+0.7$}

Examination of the Hi data around G85.4+0.7 has revealed a large feature centred at a radial velocity of $v_{\mathrm{LSR}} \approx$ $-12 \mathrm{~km} \mathrm{~s}^{-1}$ (see Fig. 7). The spatial coincidence is so good that it seems very likely that we are seeing a stellar wind bubble generated by the progenitor of the SNR, and quite possibly by other stars which are (or were) members of the
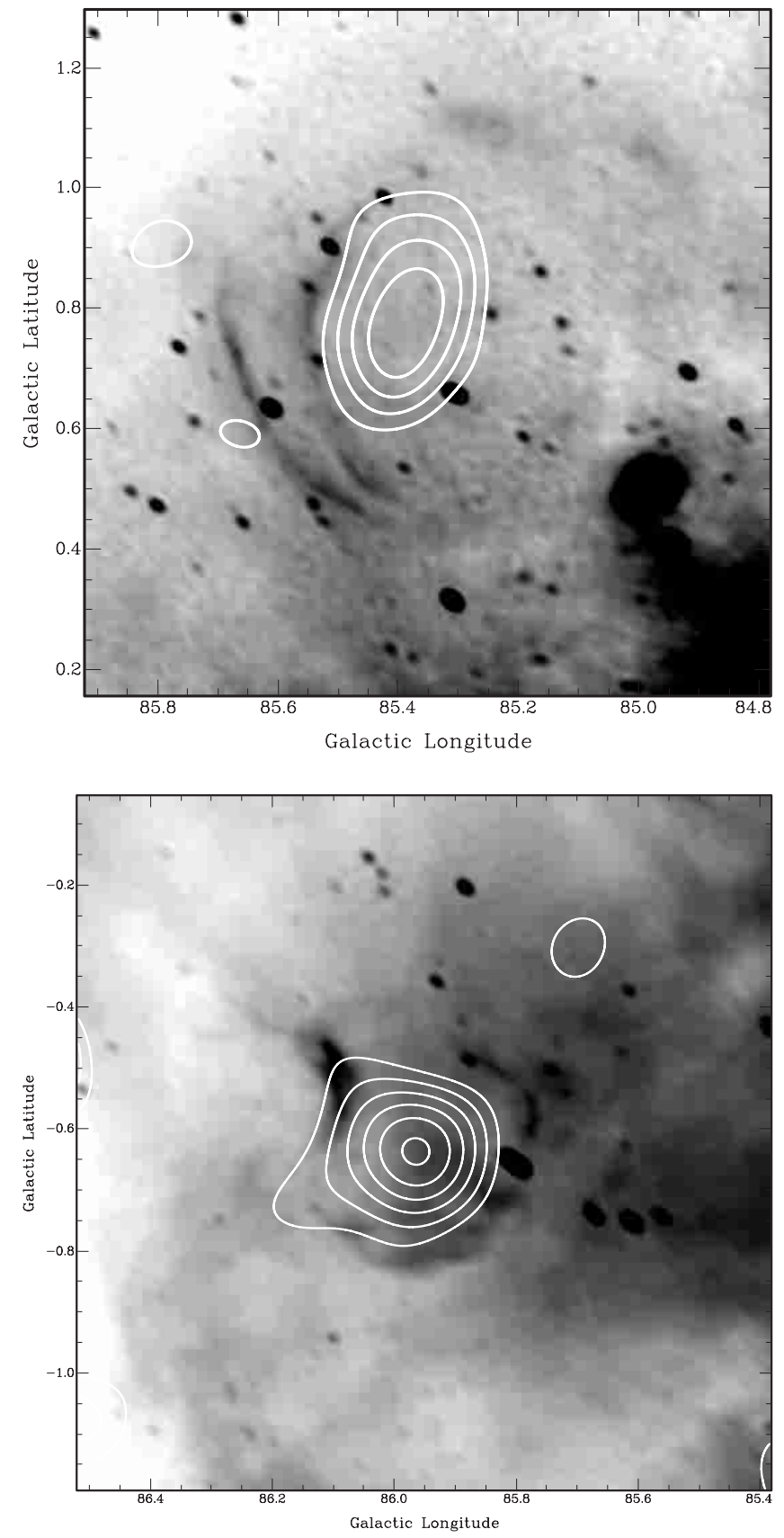

Fig. 6. Greyscale images of the new SNR G85.4+0.7 (top) and the new SNR G85.9-0.6 (bottom) at $1420 \mathrm{MHz}$. White contours represent hard X-rays (arbitrary units) taken from the ROSAT All-sky Survey.

same association. This conclusion is based on morphological resemblance only, since no expansion of the $\mathrm{H}$ i bubble can be detected in our data.

The velocity of the $\mathrm{H}$ I shell translates to a distance of $3.8 \mathrm{kpc}$ using a flat rotation model with a solar distance of $R_{\odot}=8.5 \mathrm{kpc}$ and a velocity of $v_{\odot}=220 \mathrm{~km} \mathrm{~s}^{-1}$. The H I bubble has an angular diameter of about $1.5^{\circ}$ which corresponds to $100 \mathrm{pc}$ at the proposed distance.

To determine the mass of the $\mathrm{H}$ i bubble we removed all extended background emission from all channels of our $\mathrm{H} \mathrm{I}$ data cube by subtracting a twisted plane. We calculated 


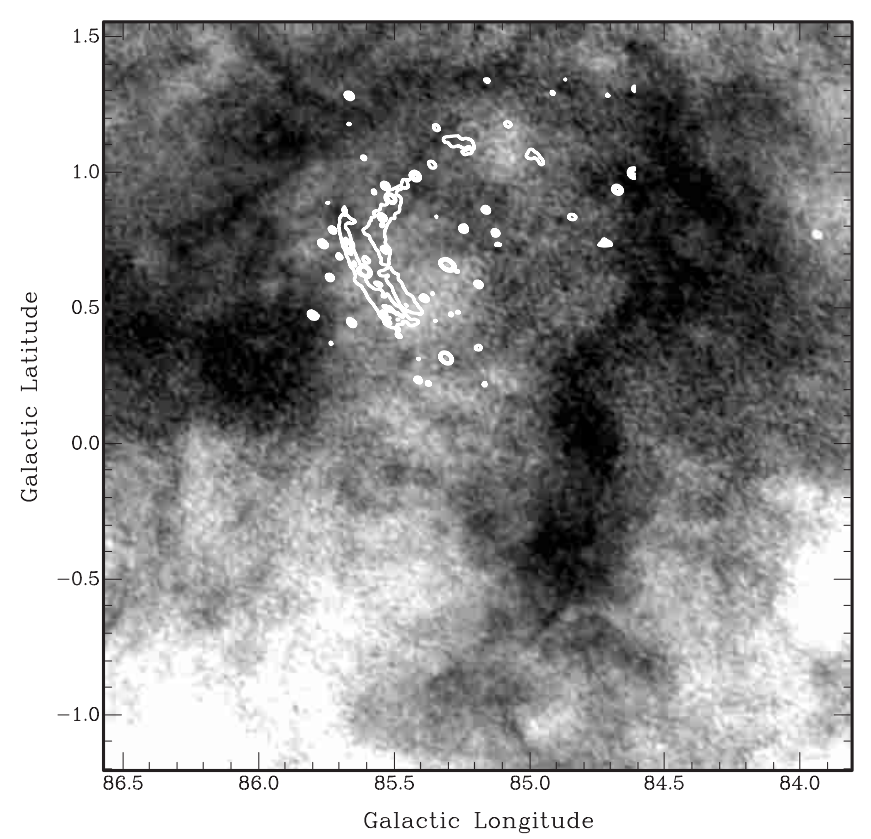

Fig. 7. Greyscale images of the atomic hydrogen around the new SNR G85.4+0.7 at $-12.18 \mathrm{~km} \mathrm{~s}^{-1}$. In this channel the stellar wind bubble is most prominent. The white contours represent the $1420 \mathrm{MHz}$ radio continuum emission.

this twisted plane by a least square fit to the edges of all channel maps. We then calculated the mean value in each channel. In Fig. 8 this mean value is plotted as a function of the radial velocity for the $\mathrm{H}$ I gas in the local spiral arm. The two major structures in this area show a Gaussian distribution as a function of radial velocity. We fitted Gaussians to these structures to separate them and to determine the total mass of the stellar wind bubble. The total mass is about $16000 M_{\odot}$. This translates to a mean density of $1.2 \mathrm{~cm}^{-3}$ around the stars before the bubble was formed. The width of the Gaussians indicates an upper limit for the expansion velocity of $6 \mathrm{~km} \mathrm{~s}^{-1}$.

\subsubsection{The $\mathrm{H}$ I environment of $\mathrm{G} 85.9-0.6$}

Inspection of the H I data in the area around G85.9-0.6 shows no structures associated with the SNR.

\subsection{The stars within the $\mathrm{HI}$ bubble around $\mathrm{G} 85.4+0.7$}

It is very unlikely that a $16000 M_{\odot} \mathrm{H} \mathrm{I}$ bubble was formed by the progenitor star of the SNR alone. Such structures are usually created by the combined stellar wind output of an $\mathrm{OB}$ association and the subsequent supernova explosions of the $\mathrm{O}$ and early B-type stars. It is very likely that some of these stars are still there since we have found thermal filaments in the area. The optical observations described in Sect. 2.2 were designed to find those stars. A $V$-band image of this observation is displayed in Fig. 9.

Reddening for each target star was interpolated from tabulated data on stellar colours and temperatures for

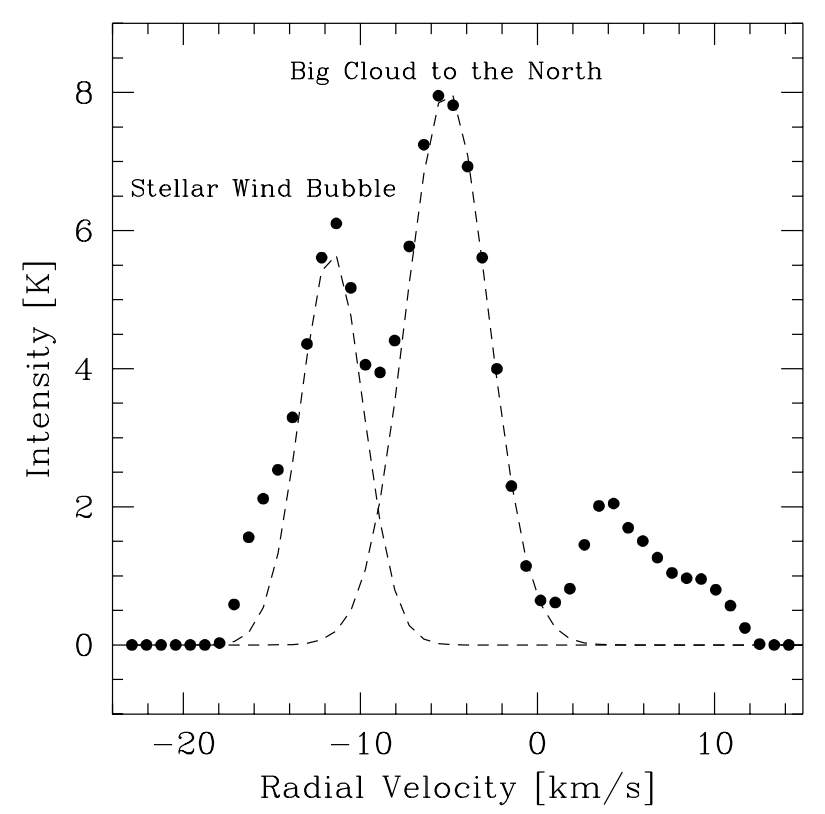

Fig. 8. Mean brightness temperature in the channels of our $\mathrm{H} \mathrm{I}$ data cube as a function of the channel number. The channels are about $0.82 \mathrm{~km} \mathrm{~s}^{-1}$ wide. The peak of our Hi bubble is at $-12.18 \mathrm{~km} \mathrm{~s}^{-1}$. The dashed lines represent Gaussians fitted to the major structures in this area.

Table 3. Observed properties of two candidate stars for association with HI stellar wind bubble.

\begin{tabular}{ccc}
\hline & Star 1 & Star 2 \\
\hline \hline$\alpha, \delta$ & $20^{\mathrm{h}} 50^{\mathrm{m}} 37^{\mathrm{s}} .8$ & $20^{\mathrm{h}} 51^{\mathrm{m}} 01^{\mathrm{s}} .3$ \\
$(\mathrm{~J} 2000)$ & $45^{\circ} 10^{\prime} 59^{\prime \prime} .8$ & $45^{\circ} 09^{\prime} 40^{\prime \prime} .2$ \\
$V$ & 12.59 & 13.28 \\
$(B-V)_{0}$ & -0.27 & -0.285 \\
$(U-B)_{0}$ & -0.945 & -1.00 \\
$E_{B-V}$ & 1.048 & 1.30 \\
$d(\mathrm{kpc})$ & $3.4 \pm 0.6$ & $3.9 \pm 0.7$ \\
Spectral Type $^{*}$ & $\mathrm{~B} 1 \mathrm{~V}$ & $\mathrm{~B} 1 \mathrm{~V}$ \\
& & \\
\hline
\end{tabular}

${ }^{*}$ Spectral types are derived from dereddened colour indices for main sequence stars.

normal stars (Allen 1976). Interstellar extinction towards this part of the Milky Way was found by Straizys et al. (1989) to be high, due to a foreground dust cloud at $550 \pm 100$ pc. Our large colour-excesses $E_{B-V}$ for the distant blue stars investigated here confirm that. Of the 45 stars investigated, two were found with derived dereddened colours consistent with stars of spectral type B1 $V$, at distances of $3.4 \pm 0.6$ and $3.9 \pm 0.7 \mathrm{kpc}$ (see Table 3 ). They are located within 4.4 arcmin of each other and are most likely members of the association responsible for the formation of the stellar wind bubble seen at a kinematic distance $3.8 \mathrm{kpc}$. 


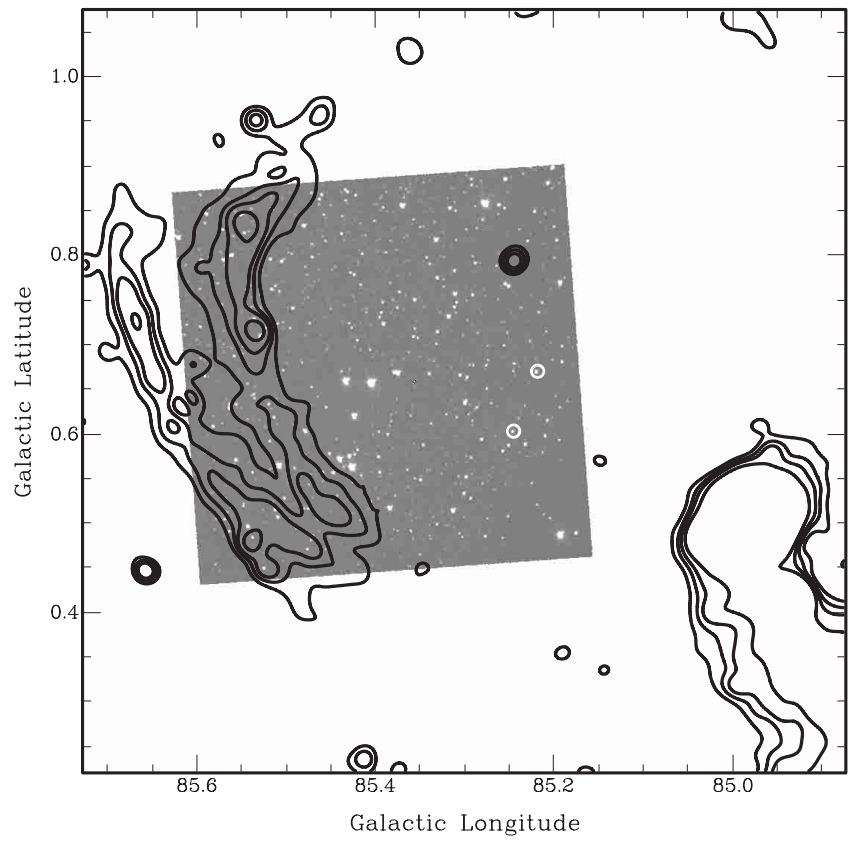

Fig. 9. $V$-band image of the centre of the $\mathrm{H}$ i bubble around G85.4+0.7 with overlaid contours representing the radio continuum emission at $1420 \mathrm{MHz}$. The position of two stars considered to be inside the stellar wind bubble is indicated.

\section{Discussion}

\subsection{The new SNR G85.4+0.7}

The supernova explosion which led to the SNR G85.4+0.7 seems to have occurred in a stellar wind bubble at a distance of about $3.8 \mathrm{kpc}$. The bubble contains approximately $16000 M_{\odot}$ of atomic hydrogen in a wide shell with a diameter of $100 \mathrm{pc}$. The shell is open to the south, most probably because of the lack of material in that direction. This bubble was probably created by the combined stellar winds of the members of an $\mathrm{OB}$ association and the subsequent explosions of the more massive stars.

With the sensitivity of our optical observations, we have detected two B1 stars within the bubble. With this sensitivity we would have been able to detect any more massive stars remaining from the original association, and we can therefore conclude that the O-type and B0 stars have already exploded. It is therefore reasonable to assume that the progenitor of G85.4+0.7 was an early B-type star.

The expanding shock wave of the supernova remnant has not yet reached the border of the Hi bubble. This explains the low radio surface brightness and the weak thermal X-ray emission, since those depend strongly on interaction with the ambient medium. Because of the low density inside the stellar wind bubble, the supernova remnant is probably still expanding freely, which means with constant velocity. An early B star has a relatively weak stellar wind, and retains most of its mass until it explodes. The ejecta might then contain up to $10 M_{\odot}$ of material. Using the canonical value of $10^{51} \mathrm{erg}$ for the explosion energy, we calculate an expansion velocity of about $3200 \mathrm{~km} \mathrm{~s}^{-1}$. At a distance of $3.8 \mathrm{kpc}$ the radius of the nonthermal shell translates to about $15 \mathrm{pc}$. The age of the SNR is then about $6300 \mathrm{yrs}$.

\subsection{The new SNR G85.9-0.6}

We do not have enough information about G85.9-0.6 to draw strong conclusions. The low radio surface brightness and the weak thermal X-ray emission again indicate a low-density ambient medium, and our failure to detect $\mathrm{HI}$ features associated with the SNR corroborates this. In this direction the line of sight passes along the local spiral arm, and we detect $\mathrm{HI}$ emission within the arm at virtually every distance to $4.5 \mathrm{kpc}$, none of it plausibly associated with the SNR. Perseus arm gas begins at $5.5 \mathrm{kpc}$, and again we see no features which we can link to the SNR. We suggest that G85.9-0.6 lies in the interarm region at about $5 \mathrm{kpc}$, in the region between the local and Perseus arm. At that distance the SNR has a diameter of about $35 \mathrm{pc}$. In the interarm region a supernova explosion of type Ia is the most likely type. In a type Ia event the mass of the ejecta is $1.4 M_{\odot}$. Assuming an explosion energy of $10^{51} \mathrm{erg}$ we would get an expansion velocity of about $8500 \mathrm{~km} \mathrm{~s}^{-1}$ and an age of about $2000 \mathrm{yrs}$ if the SNR is still expanding freely. An ambient density of less than $0.02 \mathrm{~cm}^{-3}$ is required for this. For an ambient density of $0.1 \mathrm{~cm}^{-3}$ we would get an adiabatic expanding SNR with an age of $\sim 5500$ yrs and a current expansion velocity of $\sim 1200 \mathrm{~km} \mathrm{~s}^{-1}$.

\section{Summary}

We have discovered two new SNRs of low surfacebrightness using the radio-continuum data from the CGPS. The presence of X-ray emission coincident with the radio objects confirms our interpretation. The surface brightness of these SNRs is so low that integrated flux densities cannot be obtained.

The particular properties of this survey, good angular resolution, wide angular coverage, and good sensitivity to extended emission at low radio frequencies, are needed to separate such objects from the abundance of confusing thermal emission in the Galactic plane. Good angular resolution is also needed to distinguish between weak extended emission and chance clusters of extragalactic sources.

One of the SNRs, G85.4+0.7, has occurred in a stellarwind bubble, and is probably the result of a type II SN event. Its low brightness can be interpreted as a consequence of the rarefied environment, rather than age. The other SNR, G85.9-0.6, cannot be associated with ISM features, and is probably the result of a type 1a event in the interarm region. The available evidence suggests that the two SNRs have ages of 2000 to 6000 years.

Further systematic searches of the kind we have described will be useful in testing the hypothesis that the largest fraction of SNRs are of low surface brightness, and that many young SNRs present this appearance at radio wavelength. 
Acknowledgements. The Dominion Radio Astrophysical Observatory is a National Facility operated by the National Research Council. The Canadian Galactic Plane Survey is a Canadian project with international partners, and is supported by the Natural Sciences and Engineering Research Council (NSERC). This research has made use of the ROSAT All-sky survey data which have been processed at Max-Planck-Institut für extraterrestrische Physik (MPE) in Garching (Germany). TF has been supported by a scholarship from NSERC. DL has been supported by a grant from NSERC. We wish to thank Charles Kerton for critical reading of the paper.

\section{References}

Allen, C. W. 1976, Astrophysical Quantities (Third Edition) (The Athlone Press, University of London)

Busser, J.-U., Egger, R., \& Aschenbach, B. 1996, A\&A, 310, L1

Condon, J. J., Broderick, J. J., \& Seielstad, G. A. 1989, AJ, 97,1064

Condon, J. J., Broderick, J. J., Seielstad, G. A., Douglas, K., \& Gregory, P. C. 1994, AJ, 107, 1829

Foster, T., \& Routledge, D. 2001, A\&A, 367, 635

Fürst, E., Reich, W., Reich, P., \& Reif, K. 1990, A\&AS, 85, 691

Green, D. A. 2000, A Catalogue of Galactic Supernova Remnants (August 2000 version at http://www.mrao.cam.ac.uk/surveys/snrs)
Greiner, J., Egger, R., \& Aschenbach, B. 1994, A\&A, 286, L35 Haslam, C. G. T., Stoffel, H., Salter, C. J., \& Wilson, W. E. 1982, A\&AS, 47, 1

Henden, A. A., \& Kaitchuck, R. H. 1982, Astronomical Photometry (Van Nostrand Reinhold Company, New York)

Higgs, L. A., \& Tapping K. F. 2000, AJ, 120, 2471

Kothes, R., Fürst, E., \& Reich, W. 1998, A\&A, 331, 661

Landecker, T. L., Dewdney, P. E., Burgess, T. A., et al. 2000, A\&AS, 145, 509

Landolt, A. U. 1983, AJ, 88, 439

Matthews, H. E., Baars, J. W. M., Wendker, H. J., \& Goss, W. M. 1977, A\&A, 55, 1

Oja T. 1996, Baltic Astron., 5, 103

Pfeffermann, E., Aschenbach, B., \& Predehl, P. 1991, A\&A, 246, L28

Reich, W., Fürst, E., \& Arnal, E. M. 1991, A\&A, 256, 214

Reich, W., Reich, P., \& Fürst, E. 1990, A\&AS, 83, 539

Rengelink, R. B., Tang, Y., de Bruyn, A. G., et al. 1997, A\&AS, 124,259

Sofue, Y., \& Reich, W. 1979, A\&AS, 38, 251

Straizys, V., Goldberg, E. P., Meistas, E., \& Vansevicius, V. 1989, A\&A, 222, 82

Taylor, A. R., Wallace, B. J., \& Goss, W. M. 1992, AJ, 103, 931

Taylor, A. R., Dougherty, S. M., Gibson, S. J., et al. 2001, AJ, submitted

van den Bergh, S., \& McClure, R. D. 1994, ApJ, 425, 205

Willis, A. G. 1999, A\&AS, 136, 603 